\title{
Usefulness of microvolt T-wave alternans testing in the assessment of all-cause mortality and life-threatening ventricular arrhythmia risk in patients with left ventricular dysfunction
}

\author{
Ludmiła Daniłowicz-Szymanowicz ${ }^{1}$, Małgorzata Szwoch ${ }^{1}$, Alicja Dąbrowska-Kugacka ${ }^{1}$, \\ Maria Dudziak², Dariusz Kozłowski ${ }^{1}$, Grzegorz Raczak ${ }^{1}$
}

\begin{abstract}
1Department of Cardiology and Electrotherapy, Medical University of Gdansk, Gdansk, Poland

2Department of Noninvasive Cardiac Diagnostics, Medical University of Gdansk, Gdansk, Poland
\end{abstract}

Submitted: 20 June 2013

Accepted: 23 September 2013

Arch Med Sci 2015; 11, 5: 945-951

DOI: $10.5114 /$ aoms.2013.37936

Copyright $\odot 2015$ Termedia \& Banach

\section{Abstract}

Introduction: Patients with left ventricular ejection fraction (LVEF) $\leq 35 \%$ are eligible for implantable cardioverter-defibrillator (ICD) placement in the primary prevention of sudden cardiac death. Nevertheless, other risk factors facilitating the selection of individuals with highest mortality are still sought. The aim of the study was to verify the usefulness of microvolt T-wave alternans (MTWA) testing in the assessment of all-cause mortality and life-threatening ventricular arrhythmias (EVENTs) in these patients. Previous data from the literature are inconclusive.

Material and methods: Patients with LVEF $\leq 35 \%$ were eligible if they did not have a history of sustained ventricular arrhythmias, and were treated with $\beta$-blockers. Participants underwent MTWA testing and were subsequently followed.

Results: The group consisted of 139 patients. MTWA results were classified as non-negative (MTWA_non-neg) in 93 and negative (MTWA_neg) in 46 patients. During the $14.3 \pm 8.6$ months of follow-up, EVENTs were observed in 21 patients. The 1-year EVENT rate was $16.4 \%$ among MTWA_non-neg patients, and $2.6 \%$ among MTWA_neg patients $(p=0.006)$. The sensitivity of the MTWA test was $95.24 \%$, the specificity $-38.14 \%$, the positive predictive value $-21.51 \%$ and the negative predictive value $-97.83 \%$.

Conclusions: In the group of patients with left ventricular systolic dysfunction, with the exclusion of patients with the history of life-threatening ventricular arrhythmia and individuals not being on chronic $\beta$-adrenolytic therapy, the abnormal result of MTWA testing is associated with significantly increased risk of allcause mortality and life-threatening ventricular arrhythmia during 1 year of follow-up, thus identifying the individuals at the highest risk.

Key words: prognosis, microvolt T-wave alternans, left ventricular dysfunction.

\section{Introduction}

Risk stratification in patients with left ventricular dysfunction is important from the clinical point of view [1-4]. Additionally, primary prevention of sudden cardiac death (SCD) represents an important problem of modern cardiology [3, 4]. Left ventricular ejection fraction (LVEF) is a well-established clinical parameter in patients with no history of life-threatening ventricular arrhythmia episodes [5-7]. According to current standards, an

\author{
Corresponding author: \\ Ludmiła Danitowicz- \\ Szymanowicz MD \\ Department of Cardiology \\ and Electrotherapy \\ Medical University of Gdansk \\ 7 Debinki St \\ 80-952 Gdansk, Poland \\ Phone: +48 583493910 \\ Fax: +48583493920 \\ E-mail: ludwik@gumed.edu.pl
}


implantable cardioverter defibrillator (ICD) should be placed in all patients whose LVEF is equal to $35 \%$ or lower [8].

Nevertheless, other risk factors, which can be examined via non-invasive methods, are still sought in order to allow for the identification of individuals with the highest risk of SCD amongst patients with left ventricular systolic dysfunction; such patients would most benefit from ICD implantation. The parameters that were identified in previous studies dealing with this issue include numerous autonomic nervous system activity markers, the presence of late ventricular potentials in a signalaveraged electrocardiogram, and the assessment of microvolt T-wave alternans (MTWA). Amongst these tests, MTWA deserves particular attention as it is the only examination possessing a class lla indication for risk stratification of life-threatening arrhythmias; this is in contrast to other tests, which are classified as IIb [9]. Although it is considered high, the prognostic value of the MTWA test for arrhythmic episodes in patients with left ventricular systolic dysfunction differs depending on the study. Previous data from the literature are inconclusive. There are some studies in which the usefulness of MTWA testing in prognosis of life-threatening ventricular arrhythmias was not supported [10-15]. In the present study the authors tried to discuss the potential reasons behind those differences in comparison with their own results.

The aim of the study was to verify the usefulness of MTWA testing in the assessment of allcause mortality risk and the risk of life-threatening ventricular arrhythmias in a monomorphic, precisely selected group of patients with left ventricular systolic dysfunction (i.e. treated according to the current standards, excluding individuals who were not administered $\beta$-adrenolytics on a chronic basis, and patients with a history of life-threatening ventricular arrhythmia) during an approximately 1-yearlong follow-up period.

\section{Material and methods}

\section{Patient selection}

Consecutive patients with systolic left ventricular dysfunction (LVEF $\leq 35 \%$ ) treated with $\beta$-blockers and referred to the Department of Cardiology and Electrotherapy at the Medical University of Gdansk for ICD implantation as primary SCD prophylaxis $[8,9]$ were included in the study. The exclusion criteria comprised age below 18 years, history of prior sustained ventricular arrhythmia or cardiac arrest, permanent atrial fibrillation/flutter, permanent first- or second-degree atrioventricular block, prior pacemaker implantation, New York Heart Association (NYHA) functional class IV heart failure, and the inability to exercise on a treadmill. Medical history was taken during the baseline visit, which also included physical examination and a 12-lead electrocardiogram recording. The study was performed within the framework of research on the risk stratification of life-threatening ventricular arrhythmias. The research protocol was approved by the Independent Review Board of Gdansk Medical University. Written informed consent was obtained from all participants.

\section{Microvolt T-wave alternans testing}

Microvolt T-wave alternans was tested during the treadmill exercise test. Patients were instructed to continue their current pharmacotherapy, including the use of $\beta$-blockers. Following appropriate preparation of the patients' skin in order to minimize artefacts (cleansing with abrasive paper), the electrodes were placed in three orthogonal Frank leads (X, Y, and Z; high-resolution MTWA Sensors, Cambridge Heart) as well as in 12 standard leads. The exercise test was performed on a treadmill (Delmar Reynolds) following the protocol for MTWA testing, with the heart rate gradually increasing first to $100-110$ beats per minute (bpm) and then to $110-120$ bpm (for at least $2 \mathrm{~min}$ ). Microvolt T-wave alternans was analysed using the analytic spectral method ( $\mathrm{CH} 2000$ system, Cambridge Heart, Bedford MA, USA). The computer-guided analysis was completed with an evaluation by the physician performing the test. The result of the test was classified as positive (MTWA_pos), negative (MTWA_neg) or indeterminate (MTWA_ind) on the basis of the literature-approved criteria [16, 17]. The test was considered indeterminate due to patient-related factors (inability to reach the target HR of 105$110 \mathrm{bpm}$, frequent ventricular premature beats exceeding $10 \%$ of the recording, non-sustained alternans) or to technical reasons (artefacts resulting from a high level of noise). According to the current recommendations, the test was immediately repeated in the case of any indeterminate result caused by technical reasons $[18,19]$. The patient was excluded from further analysis if the result of repeated testing was again indeterminate due to technical reasons. Based on the previous studies [20], results classified as MTWA_pos or MTWA_ind due to patient-related factors were qualified as abnormal (non-negative: MTWA_non-neg).

\section{Follow-up}

The patients were followed at the university outpatient clinic. The first visit was performed within 3 months of enrolment; subsequently, the patients were followed every 6 months or earlier if clinically required. During each visit, the clinical status of the patient was evaluated, and all adverse events were recorded. 


\section{End points}

The primary end point (EVENT) used in this study included: all-cause mortality (including SCD), nonfatal sustained ventricular arrhythmias (ventricular tachycardia (VT) or ventricular fibrillation (VF)), or ICD shocks with intracardiac electrograms documenting rapid ventricular arrhythmia (VT $\geq 170 / \mathrm{min}$ or $V F \geq 200 / \mathrm{min})$. The relevance of the intervention was verified by analysis of the electrograms stored in the memory of the ICD. Patients with more than one VT or ICD discharge reached the primary end point with the first such episode. All terminal episodes were verified with medical documentation of the patient and/or death certificate information.

\section{Statistical analysis}

The study assumed that the length of the 95\% confidence interval would not exceed $20 \%$ (that is, the error bound on the estimate would not exceed $10 \%$ ), and therefore the minimum sample size was estimated as $n=(1.96 / 0.2)^{2}=97$. For safety, in our study the sample size was set at 140 measurements. Consequently, the accuracy was improved and the error value was $8.3 \%$. We classified the MTWA test as negative or non-negative. The patients' data were censored on the date of heart transplantation, end point or last follow-up. All data are presented as the mean \pm standard deviation (SD), or as the number ( $n$ ) and percentage (\%). Due to the lack of distribution normality, the quantitative demographic and clinical data of patients from the EVENT_(+) and EVENT_(-) groups were compared using the Mann-Whitney test, while the qualitative data were compared using the $\chi^{2}$ test or Yates' $\chi^{2}$ test (depending on the sample size). The time course of the primary end point, stratified according to the MTWA results, was estimated by the Kaplan-Meier method. The association between MTWA and the primary end point was tested using the log-rank test. The event rate, estimated using the Kaplan-Meier method, was used to analyse the outcome of patients classified by MTWA. The sensitivity, specificity, positive predictive value (PPV), and negative predictive value (NPV) of MTWA for projecting end-point events during follow-up were analysed along with the $95 \%$ confidence intervals (Cls). All results were regarded as statistically significant if the $p$-values were $\leq 0.05$. The statistical analysis was conducted using the Statistica 9.0 (StatSoft, Tulsa OK, USA) software package.

\section{Results}

\section{Recruitment and baseline characteristics}

From 151 successive patients, 142 patients on $\beta$-blocker therapy were enrolled in the study; 3 subjects were later excluded because of repeated MTWA_ind tests due to technical reasons. All the patients were recruited as outpatients. The mean age of the enrolled 139 patients was $64 \pm 12$ years old, $86 \%$ of them were male, and the average LVEF was $28 \pm 7 \%$. All patients were treated with betablockers and all of them remained on their usual, long-term medications, including $\beta$-blockers, during MTWA testing.

\section{Microvolt T-wave alternans results}

Microvolt T-wave alternans results were classified as positive in 62 patients (45\%), indeterminate in 31 patients (22\%), and negative in 46 patients (33\%). The causes of MTWA_ind were as follows: inability to reach a target $H \bar{R}$ of $105-110 \mathrm{bpm}(n=19)$, frequent ventricular premature beats exceeding $10 \%$ of the recording $(n=11)$, and non-sustained alternans $(n=1)$.

\section{Primary outcome}

During the $14.3 \pm 8.6$ months of follow-up, the primary end point was observed in 21 patients. The distribution of the end-point episodes is presented in Table I. There were no demographic or clinical differences between the EVENT_(+) and EVENT_(-) groups (Table II). The 1-year event rate was $16.4 \%$ (8.1-24.0\%) among MTWA_non-neg patients, and $2.6 \%(0.0-7.4 \%)$ among MTWA_neg patients $(p=0.006)$ (Figure 1).

Table I. Distribution of clinical events contributing to the primary end points

\begin{tabular}{|lccccc|}
\hline Variable & MTWA_pos & MTWA_ind & MTWA_non-neg & MTWA_neg & Total \\
\hline No. of patients & 62 & 31 & 93 & 46 & 139 \\
\hline Sudden cardiac death & 2 & 1 & 3 & - & 3 \\
\hline Spontaneous sustained VT/VF & 2 & 1 & 3 & - & 3 \\
\hline Appropriate ICD discharge & 8 & 2 & 10 & - & 10 \\
\hline Cardiac non-arrhythmic death & 3 & - & 3 & - & 3 \\
\hline Non-cardiac death & 1 & - & 0 & 1 & 2 \\
\hline
\end{tabular}

MTWA_pos - Positive for microvolt T-wave alternans, MTWA_neg-negative for microvolt T-wave alternans, MTWA_ind-indeterminate for microvolt T-wave alternans, MTWA_non-neg-positive and indeterminate microvolt T-wave alternans, VTIVF-ventricular tachycardia/ventricular fibrillation, ICD - implantable cardioverter-defibrillator. 
Table II. Comparison of demographic and clinical data of patients from the EVENT_(-) and EVENT_(+) groups (data presented as means \pm SDs or numbers and percentages)

\begin{tabular}{|c|c|c|c|}
\hline Variable & EVENT_( $)(n=118)$ & EVENT_(+) $(n=21)$ & Value of $p$ \\
\hline Age [years] & $64 \pm 11$ & $65 \pm 14$ & 0.4 \\
\hline Males, $n(\%)$ & $100(85)$ & $19(90)$ & 0.7 \\
\hline History of ischaemic heart disease, $n$ (\%) & $82(69)$ & $18(76)$ & 0.2 \\
\hline LVEF (\%) & $29 \pm 6$ & $28 \pm 7$ & 0.6 \\
\hline \multicolumn{4}{|l|}{ NYHA class, $n(\%)$} \\
\hline NYHA I & $16(14)$ & $2(10)$ & 0.9 \\
\hline NYHA II & $82(69)$ & $14(67)$ & 0.8 \\
\hline NYHA III & $20(17)$ & $5(23)$ & 0.5 \\
\hline \multicolumn{4}{|l|}{ Medication, $n(\%)$} \\
\hline $\begin{array}{l}\text { Angiotensin-converting enzyme } \\
\text { inhibitors/angiotensin receptor blockers }\end{array}$ & $110(93)$ & $20(95)$ & 0.9 \\
\hline Spironolactone/eplerenone & $64(54)$ & $12(57)$ & 0.8 \\
\hline Amiodarone & $15(13)$ & $3(14)$ & 0.9 \\
\hline \multicolumn{4}{|l|}{ Comorbidities, $n(\%)$} \\
\hline Arterial hypertension & $70(59)$ & $12(57)$ & 0.9 \\
\hline Type 2 diabetes & $30(25)$ & $4(19)$ & 0.7 \\
\hline
\end{tabular}

LVEF - Left ventricular ejection fraction, NYHA - New York Heart Association.

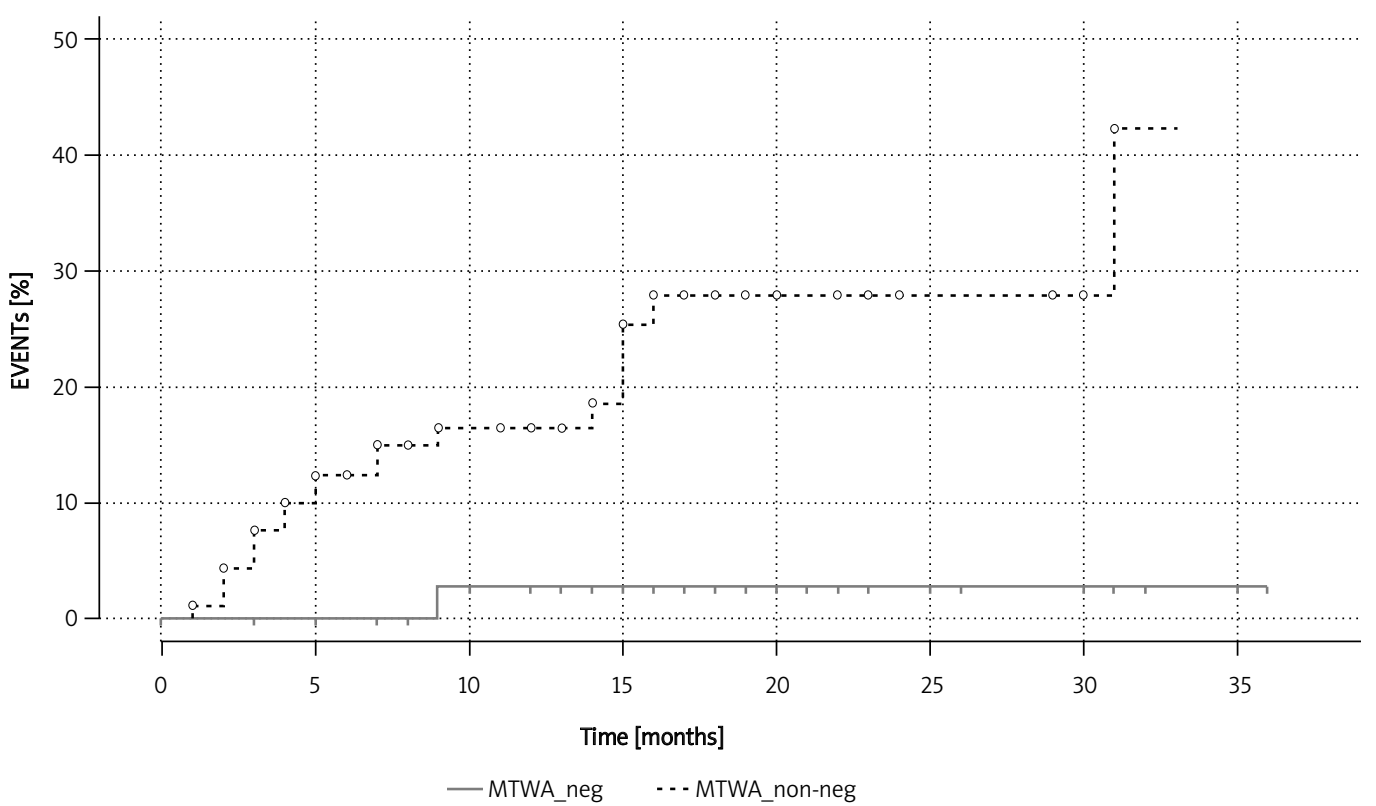
Months:
2345567891011121314151617181920212223242526
$2930313233 \quad 3536$
MTWA_neg:
$45 \quad 44413937 \quad 363432292723211917141210 \quad 9 \quad 8$
$\begin{array}{lllll}7 & 6 & 5 & 2 & 1\end{array}$
MTWA_non-neg:
92878176726660575348454139363028251916
13119
8752

Figure 1. Kaplan-Meier estimates of the probability for arrhythmic events in patients with abnormal (MTWA_non-neg) and normal (MTWA_neg) MTWA results. During the $14.3 \pm 8.6$ months of follow-up, the primary end point occurred in 20 patients with MTWA_non-neg, whereas only 1 event was documented in MTWA_neg patients $(p=0.006)$ MTWA - Microvolt T-wave alternans, MTWA_neg - negative result of MTWA testing, MTWA_non-neg - non-negative result of MTWA testing.

The sensitivity of the MTWA test was $95.24 \%$ (95\% Cl: $77.33-99.76 \%)$, the specificity was $38.14 \%$ (95\% Cl: 29.88-47.14\%), the PPV was $21.51 \%$ (95\% Cl:
$14.38-30.09 \%)$, and the NPV was $97.83 \%(95 \% \mathrm{Cl}$ : $88.66-99.89 \%)$. 


\section{Implantable cardioverter-defibrillator implantation}

All ICDs were implanted after the patients were enrolled in the study. The ICD implantation status and the reasons for ICD absence are described in Table III.

\section{Discussion}

The most important finding of our study pertains to the fact that within the monomorphic, precisely selected group of patients (i.e. treated according to the current standards, excluding individuals who were not administered $\beta$-adrenolytics on a chronic basis, and patients with a history of life-threatening ventricular arrhythmia) with left ventricular systolic dysfunction, the overall number of deaths, non-fatal VT/VF episodes, and high-voltage ICD interventions due to VT/VF during the approximately 1-year-long follow-up period was significantly higher in individuals with abnormal (nonnegative) MTWA testing than in those with a negative result of this test. In our opinion, this observation is clinically important as it allows MTWA testing to be considered as potentially useful in the selection of patients at particular risk of the abovementioned episodes. This group is regarded as a typical representation of patients qualified and waiting for ICD implantation in primary prophylaxis of sudden cardiac death. Therefore, MTWA testing performed in an appropriately selected group can be useful in identification of high- and low-risk patients.

Notably, previous studies verifying the usefulness of MTWA testing for predicting mortality risk and the risk of non-fatal arrhythmic episodes included individuals with both ischemic [10, 11, 21, 22] and non-ischemic [12, 23, 24] left ventricular systolic dysfunction. However, despite the abovementioned evidence, not all studies are consistent with the overall trend $[25,26]$.

Administration of $\beta$-adrenolytic therapy at the time of MTWA testing may be one of the most important reasons behind the discrepancies observed between the publications. For example, the MTWA_neg and MTWA_non-neg patients who participated in the MASTER trial differed significantly in terms of the frequency of $\beta$-adrenolytic use [25]. In the SCD-HeFT trial, the agent was withdrawn $24 \mathrm{~h}$ prior to the MTWA testing [26]. The results of many previously published studies suggest that the withdrawal of $\beta$-adrenolytics for the time of the examination is reflected by decreased prognostic value of the test [27-29]. For example, the metaanalysis published by Calo et al., including 15 studies of more than 5000 individuals with an average LVEF equal to $32 \%$, revealed that the negative predictive value of MTWA testing during continued
Table III. ICD implantation status

\begin{tabular}{|lcc|}
\hline \multicolumn{1}{|l}{ Variable } & MTWA_non-neg & MTWA_neg \\
\hline ICD implantation, $n$ & 79 & 28 \\
\hline \begin{tabular}{l} 
Without ICD: \\
\hline Consent not given, $n$
\end{tabular} & 5 & 6 \\
\hline $\begin{array}{l}\text { Death prior to ICD implantation: } \\
\text { SCD, } n\end{array}$ & 3 & - \\
\hline $\begin{array}{l}\text { Non-cardiac death, } n \\
\text { Awaiting ICD, } n\end{array}$ & 2 & 10 \\
\hline $\begin{array}{l}\text { Other reasons } \\
\text { Technical problems, } n\end{array}$ & - & 1 \\
\hline $\begin{array}{l}\text { Poor prognosis of } \\
\text { 1-year survival } \\
\text { (malignancy), } n\end{array}$ & - & 1 \\
\hline
\end{tabular}

ICD - Implantable cardioverter-defibrillator, SCD - sudden cardiac death, MTWA - microvolt T-wave alternans, MTWA_neg-negative results on MTWA test, MTWA_non-neg-non-negative results on MTWA test.

therapy with $\beta$-blockers is $99 \%$ as compared to $90 \%$ NPV in cases in which the agent was withdrawn for the duration of testing [29]. According to the current recommendations, MTWA assessment should be performed while patients are on their usual chronic medications, including $\beta$-blockers, as this reflects their true clinical status [17]. Our study followed this important guideline; none of the chronic pharmacotherapies (including $\beta$-adrenolytic treatment) were discontinued, and most of the studied patients used these latter agents on a long-term basis.

Duration of follow-up is another factor which could potentially influence the results of studies involving MTWA testing; longer duration of followup is associated with lower NPV [11, 30]. For example, the NPV of MTWA testing documented in the ABCD study of 566 individuals with LVEF $\leq 40 \%$ was $95 \%$ during the first year of follow-up, and decreased significantly, below $90 \%$, during the second year [11]. The duration of follow-up in the study by Grimm et al., who did not confirm the usefulness of MTWA testing in the identification of patients with increased risk of life-threatening ventricular arrhythmia or mortality, was as long as 52 months [30]. In view of the above evidence, the average follow-up of our group was 14 months.

Furthermore, the prognostic value of MTWA depends on the selection of the examined group of patients [31, 32]. For example, the NPV obtained in the course of MTWA testing was significantly reduced when studies of patients with a history of life-threatening ventricular arrhythmia were included in meta-analyses available in the literature [32]. Our present study was based on the analysis of a monomorphic, precisely selected group of patients, who had no history of previous life-threatening ventricular arrhythmia episodes, and were on 
chronic $\beta$-adrenolytic pharmacotherapy. During the approximately 1-year-long follow-up period, MTWA testing proved useful in the identification of patients who died or experienced an episode of lifethreatening ventricular arrhythmia throughout the period of the study. The authors tried to add some new information about the methodology and interpretation of MTWA testing. The main information is that MTWA testing is not useful in every group of patients, but useful in the appropriate selected patients.

The first limitation is connected with the methodology of MTWA testing, which excluded a substantial number of patients with persistent atrial fibrillation/flutter [33], atrioventricular blocks, unable to exercise on the treadmill, or in NYHA functional class IV [34]. Secondly, we did not analyse potential prognostic effects of other risk factors of life-threatening ventricular arrhythmia, such as non-sustained ventricular tachycardia, autonomic nervous system indices, or the extent of changes documented on cardiac magnetic resonance imaging. The next limitation is related to the end-point. The real problem about risk stratification remains how to identify patients at high risk of sudden cardiac death, and not all-cause mortality. The authors have accepted a composite end-point consisting of sudden cardiac death, life-threatening ventricular arrhythmias and all-cause mortality according to various literature data. However, further studies are necessary using sudden cardiac death as the primary end-point. The lack of a control group of patients without $\beta$-blockers, due to the small number of such patients, is the next limitation of the study. However, the main intention of the study was to analyse the usefulness of MTWA testing in risk stratification of patients on their chronic therapy.

Demonstrating the importance of MTWA testing for predicting mortality and non-fatal life-threatening ventricular arrhythmia has significant clinical implications. This test, if performed amongst patients with no history of VT/VF episodes, remaining on chronic $\beta$-adrenolytic therapy at the time of testing, and followed up for approximately 1 year, can be helpful in the identification of individuals at the highest risk of death or VT/VF episodes amongst subjects with left ventricular systolic dysfunction. The selection of such a group of patients seems clinically important since it reflects the characteristics of a typical population being currently qualified and subjected to ICD placement in the framework of SCD primary prevention. Microvolt T-wave alternans testing can be particularly useful in cases in which the availability of ICD placement as a means of SCD prevention is limited.

In conclusion, in a group of patients with left ventricular systolic dysfunction, treated according to the current standards, excluding individuals not on chronic $\beta$-adrenolytic therapy, and subjects with a history of life-threatening ventricular arrhythmia, an abnormal result of MTWA testing is associated with significantly increased risk of all-cause mortality and life-threatening ventricular arrhythmia during one year of follow-up, thus identifying the individuals at the highest risk.

\section{Acknowledgments}

The study was supported by a scientific grant from the Medical University of Gdansk (statute project no. ST-1, head: Prof. Grzegorz Raczak).

\section{Conflict of interest}

The authors declare no conflict of interest.

\section{References}

1. Feola M, Lombardo E, Testa M, Avogardi E, Piccolo S, Vado A. Prognostic factors of mid-term clinical outcome in congestive heart failure patients discharged after acute decompensation. Arch Med Sci 2012; 8: 462-70.

2. Płońska-Gościniak E, Lipiec P, Lancelotti P, et al. Prognostic value of low-dose dobutamine stress echocardiography in patients with aortic stenosis and impaired left ventricular function. Arch Med Sci 2013; 9: 434-9.

3. Eisenberg MS, Horwood BT, Cummins RO, ReynoldsHaertle R, Hearne TR. Cardiac arrest and resuscitation. Ann Emerg Med 1990; 19: 179-86.

4. Myerburg RJ, Kessler KM, Castellanos A. Sudden cardiac death: structure, function, and time-dependence of risk. Circulation 1992; 85 (Suppl.): 12-10.

5. Bardy GH, Lee KL, Mark DB, et al. Amiodarone or an implantable cardioverter-defibrillator for congestive heart failure. N Engl J Med 2005; 352: 225-37.

6. Kelly MJ, Thompson PL, Quinlan MF. Prognostic significance of left ventricular ejection fraction after acute myocardial infarction. A bedside radionuclide study. $\mathrm{Br}$ Heart J 1985; 53: 16-24.

7. Moss AJ, Zareba W, Hall WJ, et al. Prophylactic implantation of a defibrillator in patients with myocardial infarction and reduced ejection fraction. N Engl J Med 2002; 346: 877-90.

8. McMurray JJ, Adamopoulos S, Anker SD, et al. The Task Force for the Diagnosis and Treatment of Acute and Chronic Heart Failure 2012 of the European Society of Cardiology. Eur J Heart Fail 2012; 14: 803-69.

9. Zipes DP, Camm AJ, Borggrefe M, et al. ACC/AHA/ESC 2006 guidelines for management of patients with ventricular arrhythmias and the prevention of sudden cardiac death. Europace 2006; 8: 746-837.

10. Bloomfield DM, Bigger JT, Steinman RC, et al. Microvolt T-wave alternans and the risk of death or sustained ventricular arrhythmias in patients with left ventricular dysfunction. J Am Coll Cardiol 2006; 47: 456-63.

11. Costantini O, Hohnloser SH, Kirk MM, et al. The ABCD (Alternans Before Cardioverter Defibrillator) Trial. J Am Coll Cardiol 2009; 53: 471-9.

12. Salerno-Uriarte JA, De Ferrari GM, Klersy C, et al. Prognostic: value of T-wave alternans in patients with heart failure due to nonischemic cardiomyopathy. Results of the ALPHA Study. J Am Coll Cardiol 2007; 50: 1896-904.

13. Hohnloser SH, Ikeda T, Bloomfield DM, Dabbous OH, Cohen RJ. T-wave alternans negative coronary patients 
with low ejection and benefit from defibrillator implantation. Lancet 2003; 362: 125-6.

14. Hohnloser SH, Klingenheben T, Li YG, Zabel M, Peetermans J, Cohen RJ. T-wave alternans as a predictor of recurrent ventricular tachyarrhythmias in ICD recipients: prospective comparison with conventional risk markers. J Cardiovasc Electrophysiol 1998; 9: 1258-68.

15. Rashba EJ, Osman AF, Macmurdy K, et al. Enhanced detection of arrhythmia vulnerability using T wave alternans, left ventricular ejection fraction, and programmed ventricular stimulation: a prospective study in subjects with chronic ischemic heart disease. J Cardiovasc Electrophysiol 2004; 15: 170-6.

16. Bloomfield DM, Hohnloser SH, Cohen RJ. Interpretation and classification of microvolt T wave alternans tests. J Cardiovasc Electrophysiol 2002; 13: 502-12.

17. Verrier RL, Klingenheben T, Malik M, et al. Microvolt T-wave alternans. Physiological basis, methods of measurement, and clinical utility - Consensus guideline by International Society for Holter and Noninvasive Electrocardiology. J Am Coll Cardiol 2011; 58: 1309-24.

18. Bloomfield DM, Ritvo BS, Parides MK, Kim MH. The immediate reproducibility of $\mathrm{T}$ wave alternans during bicycle exercise. Pacing Clin Electrophysiol 2002; 25: 1185-91.

19. Chow T, Gursoy S, Onufer JR, Brinkman KM, Pu W, Cohen RJ. Clinical value of repeating indeterminate microvolt T-wale alternans tests. J Am Coll Cardiol 2005; 45: 93A.

20. Kaufman ES, Bloomfield DM, Steinman RC, et al. "Indeterminate" microvolt T-wave alternans tests predict high risk of death or sustained ventricular arrhythmias in patients with left ventricular dysfunction. J Am Coll Cardiol 2006; 48: 1399-404.

21. Chow T, Kereiakes DJ, Bartone C, et al. Prognostic utility of microvolt T-wave alternans in risk stratification of patients with ischemic cardiomyopathy. J Am Coll Cardiol 2006; 47: 1820-7.

22. Bloomfield DM, Steinman RC, Namerow PB, et al. Microvolt T-wave alternans distinguishes between patients likely and patients not likely to benefit from implanted cardiac defibrillator therapy: a solution to the Multicenter Automatic Defibrillator Implantation Trial (MADIT) II conundrum. Circulation 2004; 110: 1885-9.

23. Baravelli M, Salerno-Uriarte D, Guzzetti D, et al. Predictive significance for sudden death of microvolt-level T wave alternans in New York Heart Association class II congestive heart failure patients. A prospective study. Int J Cardiol 2005; 105: 53-7.

24. De Ferrari GM, Sanzo A. T-wave alternans in risk stratification of patients with nonischemic dilated cardiomyopathy: can it help to better select candidates for ICD implantation? Heart Rhythm 2009; 6: S29-35.

25. Chow T, Kereiakes DJ, Onufer J, et al. Does microvolt T-wave alternans testing predict ventricular tachyarrhythmias in patients with ischaemic cardiomyopathy and prophylactic defibrillators? The MASTER Trial. J Am Coll Cardiol 2008; 52: 1607-15.

26. Gold MR, Ip JH, Costantini O, et al. Role of microvolt T-wave alternans in assessment of arrhythmia vulnerability among patients with heart failure and systolic dysfunction. Circulation 2008; 118: 2022-8.

27. Chan PS, Gold MR, Nallamothu BK. Do beta-blockers impact microvolt T-wave alternans testing in patients at risk for ventricular arrhythmias? A meta-analysis. J Cardiovasc Electrophysiol 2010; 21: 1009-14.

28. Zacks ES, Morin DP, Ageno S, et al. Effect of oral betablocker therapy on microvolt T-wave alternans and electrophysiology testing in patients with ischemic cardiomyopathy. Am Heart J 2007; 153: 392-7.
29. Calo L, De Santo T, Nussio F, et al. Predictive value of microvolt T-wave alternans for cardiac death or ventricular tachyarrhythmic events in ischemic and nonischemic cardiomyopathy patients: a meta-analysis. Ann Noninvasive Electrocardiol 2011; 16: 388-402.

30. Grimm W, Christ M, Bach J, Muller HH, Maisch B. Noninvasive arrhythmia risk stratification in idiopathic dilated cardiomyopathy. Results of the Marburg Cardiomyopathy Study. Circulation 2003; 108: 2883-91.

31. Gupta A, Hoang DD, Karliner L, et al. Ability of microvolt T-wave alternans to modify risk assessment of ventricular tachyarrhythmic events: a meta-analysis. Am Heart J 2012; 163: 354-64.

32. Gehi AK, Stein RH, Metz LD, Gomes JA. Microvolt T-wave alternans for the risk stratification of ventricular tachyarrhythmias events. J Am Coll Cardiol 2005; 46: 75-82.

33. Shizuta S, Ando K, Nobuyoshi M, et al. Prognostic utility of T-wave alternans in a real-world population of patients with left ventricular dysfunction: the PREVENT-SCD study. Clin Res Cardiol 2011; 101: 89-99.

34. Jackson CE, Myles RC, Tsorlalis IK, et al. Profile of microvolt T-wave alternans testing in 1003 patients hospitalized with heart failure. Eur J Heart Failure 2012; 14: 377-86. 